\title{
WHEELS COATING PROCESS MONITORING IN THE PRESENCE OF NUISANCE PARAMETERS USING SEQUENTIAL CHANGE-POINT DETECTION METHOD
}

\author{
Karim Tout, Florent Retraint and Rémi Cogranne \\ LM2S - ICD - UMR 6281 STMR CNRS - Troyes University of Technology (UTT) - Troyes - France. \\ E-mail : \{karim.tout, florent.retraint, remi.cogranne\}@utt.fr
}

\begin{abstract}
The paper addresses the problem of monitoring a nonstationary online process to detect an abrupt failure. The process studied in this paper is the one of wheels coating, but the proposed method can be extended to a broad range of processes. Using a camera, a picture of every wheel is captured for traceability. This image is used, in our problem, to measure the coating intensity via pixels mean value. In our operational context, it is wished to control the false alarm probability over a long period (typically a day) as well as to keep the detection delay under a given number of observations, which corresponds to a small number of wheels with defective coating. The problem of abrupt coating problem detection is addressed using a sequential method that takes into account those two requirements while it is also able to adapt to the non-stationnarity of the process. Numerical results on a large set of wheels images show the efficiency of the proposed approach.
\end{abstract}

Index Terms - Industrial monitoring systems, Hypothesis testing theory, Sequential detection, Parametric model.

\section{INTRODUCTION}

In recent years, the change-point detection topic has been receiving increasing attention in various domains. It addresses the problem of detecting the point or multiple points at which a "significant change" occurs in a time series. These points are referred to as change points. The change-point detection process must be able to distinguish between a "significant change" indicating an abnormal event, and an "insignificant change" due to noise and that indicates a predicted or a normal behavior of data. Distinguishing change points from spurious noise is very important in order to keep a false alarm rate. However, surprisingly sequential methods are hardly provided with established, or bounded, false-alarm probability and power function.

In general, change-point detection methods can be classified into "posteriori" and "sequential" methods. The choice

This work has been financially supported by the Conseil Régional de Champagne-Ardenne through the project VISION. of the appropriate class of methods depends heavily on the application.

"Posteriori" methods, also referred to as offline or retrospective methods, are considered in many applications, such as climate change detection [1], biological applications [2] and analysis of social media [3], to cite few topics. This type of method is used after the reception of data with the goal to detect all the change points along and estimate their locations while not taking any corrective actions.

On the opposite, many other applications analyze data in real time with the goal to take an immediate response as soon as a change in data is detected, as it can reveal a system failure which must be handled. Such context falls within the problem of "sequential" methods, also referred to as online or real-time methods, in which it is assumed that the data is received sequentially, and that until a change point is detected the process is allowed to continue. Contrariwise, when the data changes it is aimed at detecting the change point with minimal delay time, in order to take the relevant actions, while also preserving a low false-alarm. Obviously, minimizing the detection delay and the false-alarm rate are contradictory goals. This type of method has been especially attracting attention from the industrial world, in which the term control chart is widely used, for quality control applications $[4,5,6]$.

"Sequential" change-point detection methods can be further categorized into the "parametric" and "non-parametric" methods.

On the one hand, "non-parametric" or data-driven methods have the advantages not to require any assumptions or any model on the data. They are based on statistical methods, especially supervised or non-supervised learning, to build detection rules based on large set of observations. Such decision rules are then applied to new data. While not requiring any model on the observations, those methods may, however, be limited, typically when the manufacturing process can widely change, and they are hardly provided with known statistical performances.

On the other hand, "parametric" methods are used when a sufficient information on the monitoring process is available such that a statistical model of the observation can be designed.

In other words, this approach requires that some distribu- 
tional knowledge of the data is available and employed into the detection scheme. A common limitation of these methods is that they rely on pre-specified parametric models, and based on a priori information about the form of data distribution.

The present paper falls within the scope of parametric sequential with the goal monitoring wheels paint coating process in real time in order to detect an abrupt change. In fact the coating may fail because of lack of paint or blocked spray nozzle. In this operational context, the maximal detection delay (number of wheels with coating defect) is set and it is wished to control the false-alarm probability over a fixed run length (typically a day).

The present paper is organized as follows. Section 2 presents the problem of paint coating intensity variation on produced wheels. Section 3 recalls the well-known cumulative sum (CUSUM) procedure [7] and presents the proposed linear parametric model to deal with the observations' nonstationnarity. The hypothesis test and the decision rule of the proposed method are also presented in Section 3. Section 4 presents numerical results obtained on a wide range of real images and studies the performance of the proposed method. Finally, Section 5 concludes the paper.

\section{PAINT COATING INTENSITY}

Wheel paint has two purposes; to protect the underlying metal from the harsh environment to which it is exposed, and most importantly to improve the look of wheels. Modern wheel coating methods consist of five main steps, starting with the pretreatment which removes and cleans excess metal to form a smooth surface structure, and ending with the topcoats which provide surface properties including color, appearance, gloss, smoothness, and weather resistance [8]. This paper focuses on the topcoats as they are the only visible layer.

Wheel topcoats are usually composed of several layers of paint coatings, with a precise thickness, spread on the whole surface of the wheel one after another [9].The appearance (color, gloss, texture, etc....) of a coated surface greatly affects perception on the product quality. Customers' specifications on wheels appearance are hence important and any deviation from those specifications must be detected. However, it is important to note that in this context a defective process will not only affect one wheel, but all of the following products. Therefore, a fast and accurate detection of any anomaly, as soon as it appears, is necessary in order to reduce the number of defective products, thus reducing the loss. Moreover, small deviations in the coating intensity is likely to remain unnoticed by the visual inspection.

This lead us to the necessity of an automatic inspection system that monitors the variations of the topcoat intensity, and signal the change point with minimal delay time. The detection process has to be fast and sufficiently efficient in order to distinguish between a normal state and the anomalous state.

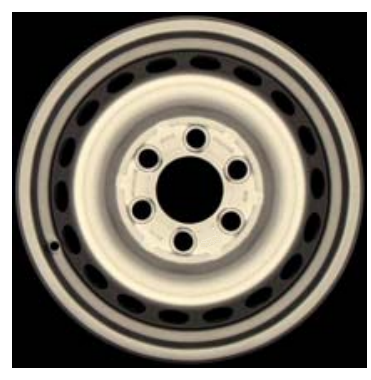

Fig. 1: An example of a wheel image

Technically speaking, many factors influence the quality of the coating, thus its appearance, such as temperature, paint viscosity, solvents, etc. $[8,9] \ldots$ This paper focuses on a usual problem, that is when the spray gun nozzle partially clogs, or gets blocked, which will be translated in a sudden change in the intensity of the topcoats.

The monitoring system consists of an imaging system placed over the conveyor belt, just after the painting process. It involves a camera that takes the image of each produced wheel, using a proper illumination setup to uniformly brighten the whole surface of the wheel while reducing light reflection artifacts. An example of a wheel image acquired by the imaging system is shown in Figure 1.

The proposed method relies on a window in the image of the wheel, over which the mean value of all pixels is computed. For one image of a wheel, let $Z=\left\{z_{w}\right\}_{w=1}^{\ell}$ denote the window containing $\ell$ pixels and $m=\ell^{-1} \sum_{w=1}^{\ell} z_{w}$ the mean value of pixels intensity. The variation of the mean value $m$ describes the variation of the topcoat intensity. Indeed, the mean value is a sufficient parameter to detect coating failure as the change in pixel values that it causes affects the whole surface of the wheel. Figure 4 shows an example of series of mean pixels value $m_{i}$, with $i$ the image index. Considering the first 1,000 images, the observed variation in the mean values is considered to be normal, and it is due to the reasons detailed previously. It is shown that the mean value of observations $m_{i}$ evolves smoothly, before or after the change. It is important to note that the variations of pixels mean is only due to the acquisition system, such as the wheel position and illumination. Based on knowledge on manufacturing processes and on observations, as illustrated in Figure 4, the process can be considered as a non-stationary process in the mean with a constant variance.

\section{SEQUENTIAL DETECTION}

\subsection{Problem Formulation}

This section formally states the problem of abrupt changepoint detection before presenting the proposed linear model of observations and the ensuing detection method.

The sequential change-point detection problem can be formulated as follows. Let us consider $\left\{X_{n}\right\}_{n \geq 1}$ a sequence of in- 
dependent and identically distributed (i.i.d) observations that is acquired sequentially. At the beginning, the sequence is considered at a normal state, and the observations follow a probability distribution $F_{\theta_{0}}(x)$. Then, at an unknown point $v \geq 0$ (the change point), the sequence reaches an abnormal state, in which the observations follow a different probability distribution $F_{\theta_{1}}(x)$. The problem formulation can be rewritten as follows:

$$
\mathbf{X}_{n} \sim\left\{\begin{array}{lll}
F_{\theta_{0}}(x) & \text { if } & 1 \leq n \leq v \\
F_{\theta_{1}}(x) & \text { if } & n \geq v+1
\end{array}\right.
$$

The sequential change-point detection consists of detecting the change point $v$ as soon as it occurs, while at the same time preserving a low false alarm rate.

For the online continuous inspection, for each new observation received, a decision rule is computed to test between the two following hypotheses:

$$
\left\{\begin{array}{l}
\mathcal{H}_{0}:\left\{\theta=\theta_{0}\right\}, \\
\mathcal{H}_{1}:\left\{\theta=\theta_{1}\right\}
\end{array}\right.
$$

As long as the test (also called stopping rule) fails to reject $\mathcal{H}_{0}$, the data acquisition continues. When the observations $x_{i}$ are statistically independent, a usual approach to decide between the hypotheses $\mathcal{H}_{0}$ and $\mathcal{H}_{1}$ is to use the cumulative sum (CUSUM) procedure which can be defined, for observations up to $N$ as follows [7]:

$$
\delta_{N}= \begin{cases}0 & \text { if } S_{1}^{N}=\max \left(S_{1}^{N-1}+s_{N}-\lambda ; 0\right)<\tau, \\ 1 & \text { if } \quad S_{1}^{N}=\max \left(S_{1}^{N-1}+s_{N}-\lambda ; 0\right) \geq \tau,\end{cases}
$$

where $\lambda$ is a constant that avoid spurious false-alarm, $\tau$ is a conveniently predefined threshold and, for initialization $S_{1}^{0}=$ 0 . Though the decision statistics $s_{N}$ and the constant $\lambda$ were not defined in [7], the logarithm of the well-known likelihood ratio is commonly used:

$$
s_{i}=\log \left(\frac{p_{\theta_{1}}\left(x_{i}\right)}{p_{\theta_{0}}\left(x_{i}\right)}\right),
$$

where $p$ is the probability density function (PDF), which is assumed to be known, and the constant $\lambda$ is usually the average of the expected values $\lambda=1 / 2\left(\mathbb{E}_{\mathcal{H}_{0}}+\mathbb{E}_{\mathcal{H}_{1}}\right)$. In the present paper, one of the most challenging problem is that the PDFs under $\mathcal{H}_{0}$ and $\mathcal{H}_{1}$ are unknown. In fact, as mentioned in Section 2, the variation of the paint coat intensity on wheels' surface is a non-stationary process in the mean, with a constant variance. Because the mean changes smoothly, it is proposed in the present paper to estimate it with a parametric linear model. In addition, in our operational context, the detection must remain below a given maximal number of defective wheels. Therefore a novel two fixed windows sequential procedure (2FW-SEQ) is proposed in this paper; the linear model parameters are estimated over the first window, and the second window is the one used for the sequential detection procedure.

\subsection{Paint Coat Intensity Model}

Let us consider a sliding window of size $L$. After the first $L$ observations, for each new received data $m_{N}$, the window slides by one point to contain the observations from $m_{N-L+1}$ to $m_{N}$. Let $Y_{N}=\left(m_{N-L+1}, \ldots, m_{N-1}, m_{N}\right)^{T}$ denotes this window after the reception of observation $m_{N}$. The vector $Y_{N}$ is modeled with the following normal distribution:

$$
\mathbf{Y}_{N} \sim \mathcal{N}\left(\mu_{N}, \sigma^{2} \mathbf{I}_{L}\right)
$$

where $\mu_{N}$ is the expectation in this window, $\mathbf{I}_{L}$ is the identity matrix of size $L$, and $\sigma^{2}$ is the variance which is assumed constant for all windows $Y_{N}, \forall N \geq L$.

A linear parametric model is proposed to represent the expectation $\mu_{N}$. It essentially consists in representing all the observations in the window $Y_{N}$ as a weighted sum of $q$ basis vectors that represent the columns of a matrix $\mathbf{H}$ of size $L \times q$. The weight of this sum represents the vector of $q$ parameters $\mathbf{d}_{N}$. Hence, the expectation $\mu_{N}$ can be written as:

$$
\mu_{N}=\mathbf{H d}_{N} .
$$

In this paper, the model of $\mathbf{H}$ is based on the following algebraic polynomial:

$$
h(x)=\sum_{j=0}^{q-1} d_{j} x^{j}
$$

with $q-1$ the degree of the polynomial.

It thus follows from Eqs. (4) and (5) that when no anomaly is present the vector of observations $Y_{N}$ is modeled by:

$$
\mathbf{Y}_{N} \sim \mathcal{N}\left(\mathbf{H d}_{N}, \boldsymbol{\sigma}^{2} \mathbf{I}_{L}\right)
$$

On the opposite, when a defect happens in the coating process, a change occurs in the mean value which will affect all observations after the change point. Consequently, when the change occurs, the observations $Y_{N}$ can be modeled as:

$$
\mathbf{Y}_{N} \sim \mathcal{N}\left(\mathbf{H d}_{N}+a \mathbf{K}_{M}, \boldsymbol{\sigma}^{2} \mathbf{I}_{L}\right),
$$

where the sudden shift in mean value is described by the vector $K_{M}$, of size $L$, containing $L-M$ zeros before the change occurs and minus ones $M$ times after, and the constant $a>0$ represents the amplitude of the change. Here, $M$ is the number of maximal acceptable wheels with defects. For example, the change vector $K_{1}=(0,0, \ldots, 0,-1)$ describes a change that only affects the last observation in the window of size $L$.

It is important to note that the "acceptable" variation of mean value, modeled by $\mathbf{H} \mathbf{d}_{N}$, is a nuisance parameter as it is of no use for the considered detection problem. To deal with this nuisance parameter, it is proposed to use the maximum likelihood (ML) estimation method to perform a rejection of this nuisance parameter as follows:

$$
\mathbf{r}_{N}=\frac{1}{\sigma} \mathbf{W} \mathbf{Y}_{N}
$$


Here $\mathbf{W}$ is the orthogonal projection of size $L-q \times L$, onto the null space of $\mathbf{H}$ whose vectors corresponds to the eigenvectors of matrix $\mathbf{I}_{L}-\mathbf{H}\left(\mathbf{H}^{\mathrm{T}} \mathbf{H}\right)^{-1} \mathbf{H}^{\mathrm{T}}$ associated with eigenvalues equal to 1 . The vector $\mathbf{r}_{N}$ represents the projection of the observations onto the null space of $\mathbf{H}$.

\subsection{FW-SEQ procedure}

Among others, the matrix $\mathbf{W}$ has the following useful properties: $\mathbf{W} \mathbf{W}^{T}=\mathbf{I}_{L-q}$; it thus follows from Eqs. (7)-(9), that the residuals $\mathbf{r}_{N}$ can be modeled under hypotheses $\mathcal{H}_{0}$ and $\mathcal{H}_{1}$ by the following statistical distribution:

$$
\left\{\begin{array}{l}
\mathcal{H}_{0}:\left\{\mathbf{r}_{N} \sim \mathcal{N}\left(\mathbf{0}, \mathbf{I}_{L-q}\right)\right\} \\
\mathcal{H}_{1}:\left\{\mathbf{r}_{N} \sim \mathcal{N}\left(\frac{a}{\sigma} \boldsymbol{\theta}_{M}, \mathbf{I}_{L-q}\right)\right\}
\end{array}\right.
$$

where $\boldsymbol{\theta}_{M}$ represents the shift of expectation, due to coating failure, projected onto the null space of $\mathbf{H}: \boldsymbol{\theta}_{M}=\mathbf{W} \mathbf{K}_{M}$.

From definition of hypotheses (10), after rejection of nuisance parameter $\mathbf{H d}_{N}$, it is obvious that the problem of detection considered essentially consists in detection of signal in noise. Similar approaches have been studied in the context of sequential detection in $[10,11]$. They proposed to use the well-known match space detection which is given in our case by:

$$
\delta_{N}=\left\{\begin{array}{lll}
0 & \text { if } & \widetilde{S}_{N-L+1}^{N}=\boldsymbol{\theta}_{M}^{T} \mathbf{r}_{N}<\tau \\
1 & \text { if } & \widetilde{S}_{N-L+1}^{N}=\boldsymbol{\theta}_{M}^{T} \mathbf{r}_{N} \geq \tau
\end{array}\right.
$$

From Eq. (10) it is straightforward to establish the statistical distribution of result $\widetilde{S}_{N-L+1}^{N}$ of the proposed $2 \mathrm{FW}-\mathrm{SEQ}$ :

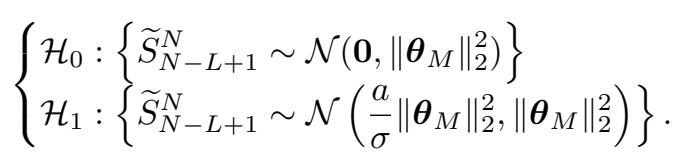

Equation (12) emphasizes the main advantages of the proposed approach. In fact, two important criteria can be used to highlight the performance of a sequential method; the false alarm probability, denoted $\alpha$, and the detection power, denoted $\beta$. For a prescribed run length, the false alarm probability depends on the given detection delay, and $\beta$ depends on the "change-to-noise ratio" $a / \sigma$ and on the detection delay $M$.

It is important to note that the choice of $L$ is crucial and must meet the following trade-off: first, $L$ must be much greater than $M$ in order to avoid the estimate of linear model parameters $\mathbf{d}_{N}$ to be impacted significantly by the abrupt change. On the opposite, $L$ must remain reasonably small such that the linear model will well model the observations' expectation and to ensure that the residuals $\mathbf{r}_{N}$ follow a standard normal distribution under $\mathcal{H}_{0}$.

\section{EXPERIMENTS AND RESULTS}

Following the constraints on the parameters $L$ and $q$, multiple simulations performed on a data base of 500000 non- anomalous observations (without change point) have led to the choice of $L=150$ and $q=3$, for which the empirical distribution of the normalized residuals $r_{n}$ shows good results compared to the theoretical one (10) in Figure 2. These simulations also helped to assure that the standard deviation has a constant value $\sigma=22$ over all the observations.

Then, it is proposed to study the effect of the second window length $M$ on the detection performances. The same data base has been used to perform a Monte-Carlo simulation, for which a simulated shift of amplitude $a=60$ has been superimposed on some of the observations. Figure 3 represents the empirical false alarm probability $\alpha$ and detection power $\beta$ over a run length $K=5000$ for 3 different values of $M=\{1,3,5\}$, as a function of the decision threshold $\tau$. When $M$ increases, $\left\|\boldsymbol{\theta}_{M}\right\|_{2}$ increases, which affects both the false alarm rate and detection power, as seen in (12). The shift between the detection power and the false alarm probability becomes larger which implies a better detection performance, but at a larger delay $M$. Depending on the application, this test allows to increase the detection power at a cost of larger detection delay, or minimize the detection delay at a cost of lower detection power.

Finally, it is wished to test the proposed sequential detection method 2FW-SEQ on a real case scenario with a real change point in the observations. Figure 4 portrays a real case of observations when the spray gun nozzle got partially clogged. As a consequence, a sudden shift in the observations can be seen at exactly the image index 2434. The blue plot represents the real observations, while the red plot represents the expectation values (5) estimated using the polynomial model over a window of size $L=150$ and a degree of $q-1=2$. Then, Figure 5 illustrates the results of the proposed 2FW-SEQ method with $M=1$ and $\tau=3.6$. It can be seen that the change point is detected at the index 2434 which means a delay of exactly 1 wheel.

Usually, when the change is detected, the sequential process stops. However, to the purpose of comparing this result with the theoretical one (12), all the results after the change has been performed on a window of size $L$ that consists of the last $L-M$ observations before the change along with the new observation as the $L^{t h}$ member. Before the change, the results show a Gaussian behavior with zero mean and a variance $\left\|\boldsymbol{\theta}_{M}\right\|_{2}^{2}=0.94$. However, after the change, the mean value undergoes a shift of $\frac{a}{\sigma}\left\|\boldsymbol{\theta}_{M}\right\|_{2}^{2}$ with $a=55$ and $\sigma=22$. The empirical results $\widetilde{S}_{i-L+1}^{i}$ before and after the change proved to be consisting with their theoretical distributions (12).

\section{CONCLUSION}

This paper proposes a method for online monitoring of a nonstationary process. The CUSUM method is modified to adapt to the operational requirements of the industrial context in order to control the false-alarm probability over a fixed run length and for a given detection delay. For the coating pro- 


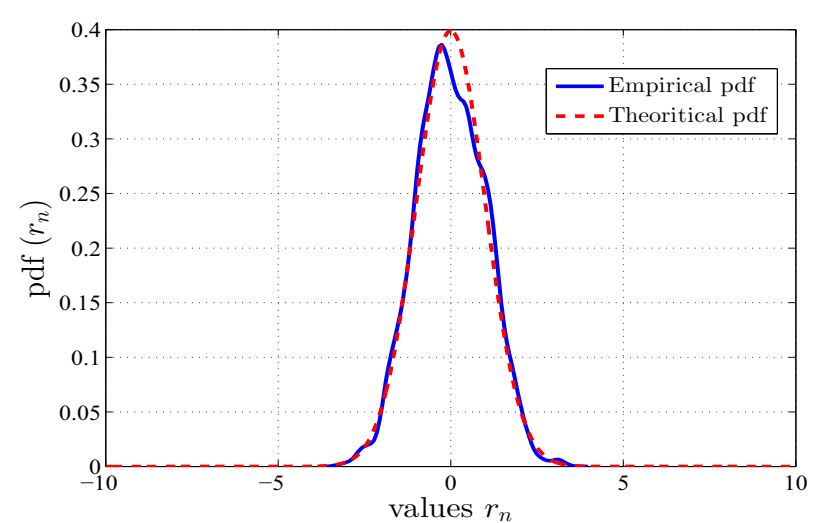

Fig. 2: Empirical and theoretical distributions of the normalized residuals $r_{n}$ for non-anomalous observations

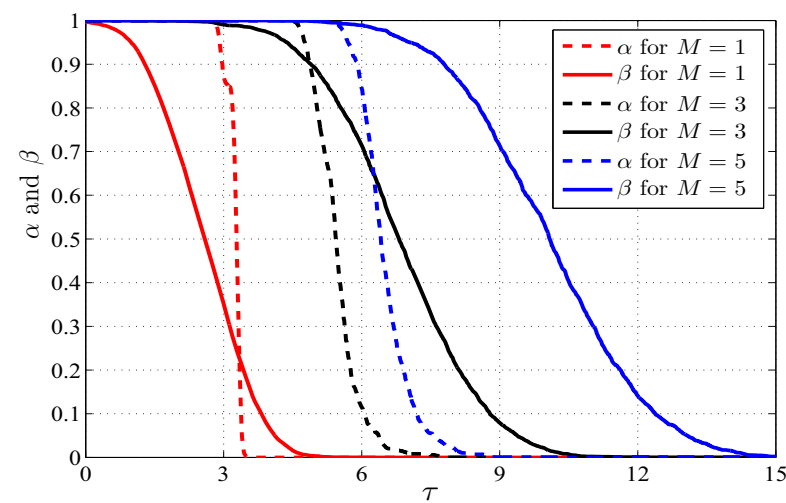

Fig. 3: Empirical false alarm probability $\alpha$ and detection power $\beta$ over a run length $K=5000$ for 3 different values of $M$, plotted as a function of the decision threshold $\tau$.

cess studied in this paper, the mean value of pixels from images of wheels are used to measure the coating intensity. As the monitoring process is non-stationary in the mean, a linear parametric model is proposed to reject this nuisance parameter. Numerical results on a large set of images show the accuracy of the proposed model and the efficiency of the proposed detection method.

\section{REFERENCES}

[1] J. Reeves, \& al. "A review and comparison of changepoint detection techniques for climate data," Journal of Applied Meteorology and Climatology, vol. 46, no. 6, pp. 900-915, 2007.

[2] G. E. Evans, \& al. "Estimating change points in biological sequences via the cross-entropy method," Annals of Operations Research, vol. 189, no. 1, pp. 155-165, 2011.

[3] A. Aprem and V. Krishnamurthy, "Utility change point detection in online social media: A revealed preference framework," IEEE Transactions on Signal Processing, vol. PP, no. 99, pp. 1-1, 2017.

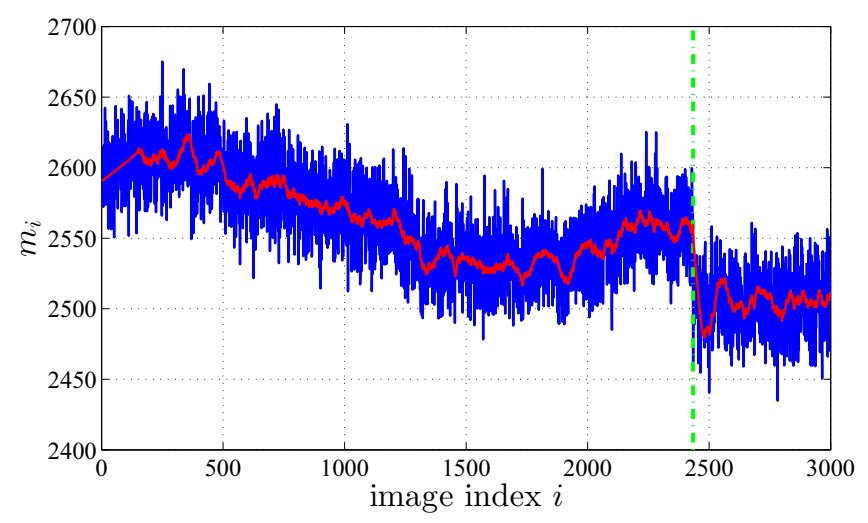

Fig. 4: Real example of the variation of mean value with a change point at index 2434 .

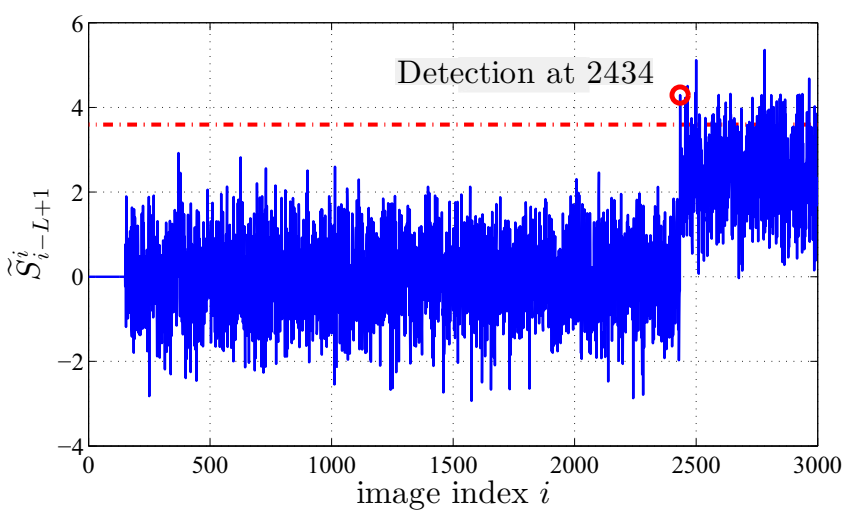

Fig. 5: Results of the proposed 2FW-SEQ with $M=1$

[4] L. H. Chiang, \& al. Fault Detection and Diagnosis in Industrial Systems, Springer London, London, 2001.

[5] A. Tartakovsky, I. Nikiforov, and M. Basseville, Sequential Analysis: Hypothesis Testing and Changepoint Detection, Chapman \& Hall/CRC Monographs on Statistics \& Applied Probability. Taylor \& Francis, 2014.

[6] H. V. Poor and O. Hadjiliadis, Quickest Detection, Cambridge University Press, 2008.

[7] E. S Page, "Continuous inspection schemes," Biometrika, vol. 41, no. 1/2, pp. 100-115, 1954.

[8] N. K. Akafuah \& al. "Evolution of the automotive body coating process-a review," Coatings, vol. 6, no. 2, 2016.

[9] N. K. Akafuah, Automotive Paint Spray Characterization and Visualization, pp. 121-165, Springer Netherlands, Dordrecht, 2013.

[10] R. Cogranne, "A sequential method for online steganalysis," in IEEE International Workshop on Information Forensics and Security (WIFS), Nov 2015, pp. 1-6.

[11] B. K. Guépié , L. Fillatre, and I .Nikiforov, "Sequential detection of transient changes," Sequential Analysis, vol. 31, no. 4, pp. 528-547, 2012. 